\title{
Influence of quantum fluctuations on zero-temperature phase transitions between collinear and noncollinear states in frustrated spin systems
}

\author{
Sven E. Krüger and Johannes Richter \\ Institut für Theoretische Physik, Universität Magdeburg, \\ P.O.Box 4120, 39016 Magdeburg, Germany
}

(Dated: October 26, 2018)

\begin{abstract}
We study a square-lattice spin-half Heisenberg model where frustration is introduced by competing nearest-neighbor bonds of different signs. We discuss the influence of quantum fluctuations on the nature of the zero-temperature phase transitions from phases with collinear magnetic order at small frustration to phases with noncollinear spiral order at large frustration. We use the coupled cluster method (CCM) for high orders of approximation (up to LSUB6) and the exact diagonalization of finite systems (up to 32 sites) to calculate ground-state properties. The role of quantum fluctuations is examined by comparing the ferromagnetic-spiral and the antiferromagnetic-spiral transition within the same model. We find clear evidence that quantum fluctuations prefer collinear order and that they may favour a first order transition instead of a second order transition in case of no quantum fluctuations.
\end{abstract}

Introduction. While quantum fluctuations do not influence the critical properties of phase transitions at $T>0$ they play an important role at $T=0$ and can yield to quantum phase transitions, which have attracted a lot of attention in recent times (see, e.g., Ref. 1). Quantum fluctuations arise due to Heisenberg's uncertainty principle and play a similar role as thermal fluctuations (for $T>0)$ in classical transitions. The spin-half Heisenberg model is a basic model which shows strong quantum fluctuations in the antiferromagnetic case. While the ground-state of the pure Heisenberg antiferromagnet (HAFM) on the square lattice shows Néel longrange order日 (LRO), a competition of bonds can increase quantum fluctuations and may result in rotationally invariant paramagnetic states, suppressing the (collinear) Néel order. This is demonstrated by recent experiments on (quasi-)two-dimensional Heisenberg systems, like $\mathrm{CaV}_{4} \mathrm{O}_{9}$ (see, e.g., Refs. 3,4) or $\mathrm{SrCu}_{2}\left(\mathrm{BO}_{3}\right)_{2}$ (see, e.g., Refs. [5.65).

Besides local singlet formation magnetic frustration is an important mechanism to drive zero-temperature transitions. In the classical Heisenberg model strong frustration often leads to noncollinear (e.g., spiral) spin states which may or may not have counterparts in the quantum case. It is generally argued that quantum fluctuations prefer a collinear ordering. A typical example is the frustrated spin-half $J_{1}-J_{2}$ model on the square lattice (see, e.g., Refs. 0,8,9,10,11). Here the classical version of the $J_{1}-J_{2}$ model has a continuously degenerate ground state for $J_{2}>J_{1} / 2$, but quantum fluctuations can remove this degeneracy yielding to a collinear state ("order from disorder" phenomenon, see, e.g., Refs. 12,13). Moreover quantum fluctuations can shift the critical point of a collinear-noncollinear transition so that the collinear state can survive into a region where classically it is already unstable.45 15

In this paper we extend our previous work, 16 where we have studied the transition from a collinear Néel order to noncollinear spiral order in a frustrated spin-half HAFM and consider now the transition form a collinear ferromagnetic order to a noncollinear spiral order within the same model. While in the classical version of the model both situations can be mapped onto each other the quantum model behaves basically different in both cases. That is because of the different nature of the collinear state: While the quantum Néel state on two-dimensional lattices exhibits strong quantum fluctuations (the sublattice magnetization of the HAFM on the square lattice is only about $60 \%$ of its classical value) the ferromagnetic state is the same for the quantum and the classical model and there are no quantum fluctuations in this state.

We use the coupled cluster method 7.18 (CCM) and exact diagonalization of finite systems to calculate the ground state. The CCM is a very powerful method and, particularly high-order implementations of this method can be used to obtain a consistent description of various aspects of quantum spin systems (for an overview see, for example, Refs. 19,20,21,22, 23,24). We note that another important method for spin systems, the quantum Monte Carlo method, cannot be used for frustrated spin systems since it suffers from the minus sign problem.

The model. We consider a spin-half Heisenberg model 
on a square lattice with two kinds of nearest neighbour bonds $J$ and $J^{\prime}$, as shown in Fig. 1,

$$
H=J \sum_{\langle i j\rangle_{1}} \mathbf{S}_{i} \cdot \mathbf{S}_{j}+J^{\prime} \sum_{\langle i j\rangle_{2}} \mathbf{S}_{i} \cdot \mathbf{S}_{j} .
$$

The sums over $<i j>_{1}$, and $<i j>_{2}$ represent sums over the nearest-neighbour bonds shown in Fig. 1 by dashed and solid lines, respectively. Each square-lattice plaquette consists of three $J$ bonds and one $J^{\prime}$ bond. A model with such a zigzag pattern has been treated with various methods. 162525

In this paper we consider only the cases in which $J$ and $J^{\prime}$ have different signs (i.e., one bond is ferromagnetic while the other is antiferromagnetic) so that the plaquettes are frustrated. The case with antiferromagnetic $J$ bonds (i.e., $J>0$ and hence $J^{\prime}<0$ ) has been studied previously using linear spin wave theory, 26 exact diagonalization and coupled cluster method 16 We therefore focus in this paper our attention mainly on the ferromagnetic case (i.e., $J<0$ and $J^{\prime}>0$ ) but compare the obtained results with those of the antiferromagnetic case.

Classical ground state. We consider the ground state of the classical version of model (1), i.e., the spins $\mathbf{S}_{i}$ are assumed to be classical vectors. For $\left|J^{\prime}\right|<|J| / 3$ (and $J$ and $J^{\prime}$ having different signs) the ground state of (11) is collinear (i.e., ferromagnetic or antiferromagnetic depending on the sign of $J$ ). At the critical point $J_{c}^{\prime}=-J / 3$, a second-order transition takes place from the collinear state to a noncollinear state of spiral nature (see Fig. 1), with a characteristic pitch angle $\Phi= \pm\left|\Phi_{\text {cl }}\right|$ given by

$$
\left|\Phi_{\mathrm{cl}}\right|=\left\{\begin{array}{ll}
0 & \left|J^{\prime}\right|<\frac{|J|}{3} \\
\arccos \left(\frac{1}{2} \sqrt{1+\frac{1}{\mid J^{\prime}}}\right) & \left|J^{\prime}\right| \geq \frac{|J|}{3}
\end{array} .\right.
$$

Note that for $\Phi=0$ this is the collinear state.

The spins $\mathbf{S}_{A}$ and $\mathbf{S}_{B}$, belonging to the $A$ and $B$ sublattices respectively, can be expressed in terms of the spiral $\mathbf{k}$ vector 16 with $\mathbf{k}=(2 \Phi, 0)$ (and see Fig. 1). We note that this spiral state is incommensurate in the $x$ direction. We also note that for the classical model the antiferromagnetic case can be transformed into the ferromagnetic case by the simultaneous substitution $J \rightarrow-J$, $J^{\prime} \rightarrow-J^{\prime}, \mathbf{S}_{i \in B} \rightarrow-\mathbf{S}_{i \in B}$. Hence the physics for both cases is classically the same.

Calculation of the quantum ground state. To calculate the quantum ground state of the Hamiltonian (11) we use the CCM. Details concerning the treatment of the model
(1) with the CCM are given in Ref. 16. We use the CCM for high orders of approximation up to LSUB6 (using 1638 fundamental configurations).

We further exactly diagonalize finite lattices of rectangular shape $\left(L_{x} \times L_{y}=4 \times 4,6 \times 4,8 \times 4\right)$ using periodic boundary conditions. The longer side $L_{x}$ of the rectangle corresponds to the direction of the $J^{\prime}$ bonds and so we can diminish the influence of the boundary conditions by an increase of $L_{x}$.

The collinear-noncollinear transition. While classically we have always a second-order phase transition from collinear order to noncollinear order at $J_{c}^{\prime}=-J / 3$, we obtain for the quantum case a different behaviour for the ferromagnetic and the antiferromagnetic case.

Using the CCM we find for the antiferromagnetic case $(J=+1)$ indications for a shift of this critical point to a value $J_{c}^{\prime} \approx-1.35$ (see Fig. (1). On the other hand for the ferromagnetic case $(J=-1)$ we do not find such a shift (see Fig. (4). The exact diagonalization (ED) data of the structure factor $S(\mathbf{k})$ (see Figs. 2 2 and 3) agree to these findings. For $J=+1$ (see Fig. 2) the collinear Néel order $[\mathbf{k}=(0,0)]$ becomes unstable against the noncollinear spiral order $[\mathbf{k}=(\pi / 4,0)]$ in the classical model for $J^{\prime} \lesssim$ -0.36 but in the quantum case only for $J^{\prime} \lesssim-0.95$. The situation for the ferromagnetic case $(J=-1)$ is again different. Here the results of the structure factor (see Fig. 3) show that the transition from $\mathbf{k}=(0,0)$ (collinear ferromagnetic order) to $\mathbf{k}=(\pi / 4,0)$ (spiral order) takes place at nearly the same value of $J^{\prime} \approx 0.36$ for both, the classical case and the quantum case.

Taking the deviation of the on-site magnetic moment $\left\langle S_{i}\right\rangle$ from its classical value $\left\langle S_{i}\right\rangle_{\mathrm{cl}}=1 / 2$ as an indication for the degree of quantum fluctuations we can compare the strength of quantum fluctuation near the collinearnoncollinear transitions for both, the antiferromagnetic and the ferromagnetic case. As reported in Ref. 16 for $J=+1$ the quantum fluctuations are particularly strong near the antiferromagnetic-spiral transition leading to an on-site magnetic moment less then $20 \%$ of its classical value. On the other hand, it can be seen from Fig. 5 that the on-site magnetic moment takes its classical value $1 / 2$ up to $J^{\prime} \approx 0.36$ for $J=-1$ and therefore virtually no quantum fluctuations occur at the ferromagneticspiral transition. Hence the shift of the critical $J_{c}^{\prime}$ in the antiferromagnetic case can clearly be attributed to the strong quantum fluctuations. In general our findings are consistent with the statement that quantum fluctuations (which we have in the antiferromagnetic case only) pre- 
fer a collinear ordering, so that in this case the quantum collinear state can survive into a frustrated region where classically the collinear state is already unstable.

We further note an agreement between the CCM results and the ED results beyond the critical $J_{c}^{\prime}$. By examining the structure factors (see Figs. 22 and 3 ) we find that for the antiferromagnetic (respectively ferromagnetic) case the transitions to a spiral state with a greater $\mathbf{k}$ vector (i.e., with a greater pitch angle $\Phi$ ) occur in the quantum model always at a greater (respectively smaller) absolute value of $J^{\prime}$ then the corresponding classical transitions. This agrees with the CCM results of the pitch angle (see Fig. 国), where we have $\Phi_{\mathrm{qm}}<\Phi_{\mathrm{cl}}$ (respectively $\Phi_{\mathrm{qm}}>\Phi_{\mathrm{cl}}$ ).

The discussion given above corresponds to our finding concerning the order of the transition. Clearly in the ferromagnetic case $\left(J^{\prime}=-1\right)$ both the classical and the quantum model show a second-order phase transition (see Figs. (1) and 5). On the other hand it was discussed in Ref. 16 that the collinear-noncollinear transition in the antiferromagnetic case $(J=+1)$ is probably a first-order

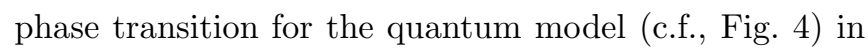
difference to the second-order transition in the classical case.

Formation of local singlets. For sufficient strong antiferromagnetic $J^{\prime}$ bonds the system (1) is characterized by a tendency to singlet pairing of the two spins coupled by a $J^{\prime}$ bond and hence the long-range magnetic (collinear or noncollinear) order is destroyed. We obtain clear indications of a second-order phase transition to a quantum paramagnetic dimerized phase at a certain critical value of $J^{\prime}=J_{s}^{\prime}$. For $J^{\prime}>J_{s}^{\prime}$ the on-site magnetic moment $\left\langle S_{i}\right\rangle$ becomes zero. For the ferromagnetic case $(J=-1)$ we find $J_{s}^{\prime} \approx 1$ using the extrapolated CCM-LSUB $n$ results (see Fig. 5). We may also consider the inflection points of $\left\langle S_{i}\right\rangle$ versus $J^{\prime}$ for the LSUB $n$ approximations, assuming that the true curve will have a negative curvature up to the critical point. We find the corresponding inflection points at $J^{\prime} \approx 1.2(n=2), J^{\prime} \approx 0.76(n=4)$ and $J^{\prime} \approx 0.74(\mathrm{n}=6)$, indicating a critical $J_{s}^{\prime}$ even slightly smaller then $J_{s}^{\prime} \approx 1$.

The ED data give a similar approximation of $J_{s}^{\prime}$ : For $J^{\prime} \approx 1$ finite-size effects in spin-spin correlations disappear almost completely (see for illustration Fig. 6). This indicates that spin-spin correlations are short ranged with a length scale smaller than the size $L_{x}$.

We note that for the antiferromagnetic case $(J=+1)$ the strength of antiferromagnetic $J^{\prime}$ needed for breaking Néel order by formation of local singlets is much larger $\left(J_{s}^{\prime} \approx 3\right.$, see Ref. 16). The lower critical $J_{s}^{\prime} \approx 1$ in the ferromagnetic case is due to frustration which assists local singlet formation (c.f., Ref. 27,28).

Summary. Using the CCM and the ED technique we have studied the influence of quantum fluctuations on zero-temperature transitions between collinear ordered and noncollinear ordered states in a frustrated spin-half square-lattice Heisenberg model with two kinds of nearest-neighbour exchange bonds. The frustration drives a second-order transition between collinear (antiferro- or ferromagnetic) and noncollinear (spiral) states in the classical model. For the quantum model the CCM provides a consistent description of collinear, noncollinear, and disordered phases, while some other standard techniques (e.g., QMC) are not applicable. We find a strong influence of quantum fluctuations on the nature of the collinear-noncollinear transition, and quantum fluctuations (which favour collinear ordering) may change the second-order classical transition to a firstorder quantum transition. If quantum fluctuations are suppressed in the collinear phase of the quantum model, the transition to the spiral phase is similar for the quantum and for the classical model.

Acknowledgments. We thank the Deutsche Forschungsgemeinschaft (Ri 615/9-1) for its support. We are indebted to J. Schulenburg for numerical assistance.
1 S. Sachdev, Quantum Phase Transitions (Cambridge University Press 1999).

${ }^{2}$ E. Manousakis, Rev. Mod. Phys. 63, 1 (1991).

${ }^{3}$ S. Taniguchi, T. Nishikawa, Y. Yasui, Y. Kobayashi, M. Sato, T. Nishioka, M. Kontani, and K. Sano, J. Phys. Soc. Jpn. 64, 2758 (1995).

4 M. Troyer, H. Kontani, and K. Ueda, Phys. Rev. Lett. 76, 3822 (1996).
5 H.Kageyama, K.Yoshimura, R.Stern, N.V.Mushnikov, K.Onizuka, M.Kato, K.Kosuge, C.P.Slichter, T.Goto, Y.Ueda, Phys.Rev.Lett. 82, 3168 (1999).

${ }^{6}$ A. Koga and N. Kawakami, Phys. Rev. Lett. 844467 (2000).

7 J. Richter, Phys. Rev. B 47, 5794 (1993).

8 J. Oitmaa and Zheng Weihong, Phys. Rev. B 54, 3022 (1996). 
9 R.F. Bishop, D.J.J. Farnell, and J.B. Parkinson, Phys. Rev. B 58, 6394 (1998).

10 V.N. Kotov, J. Oitmaa, O.P. Sushkov, and Zeng Weihong, Phys. Rev. B 60, 14613 (1999).

${ }^{11}$ L. Capriotti and S. Sorella, Phys.Rev.Lett. 84, 3173 (2000).

12 S. Prakash and C.L. Henley, Phys. Rev. B 42, 6574 (1990).

13 K. Kubo and T. Kishi, J. Phys. Soc. Jpn. 60, 567 (1991).

14 N.B. Ivanov, J. Richter, and U. Schollwöck, Phys. Rev. B 58, 14456 (1998).

15 J. Richter, U. Schollwöck, and N.B. Ivanov, Physica B 281\&282, 845 (2000).

16 S.E. Krüger, J. Richter, J. Schulenburg, D.J.J. Farnell, and R.F. Bishop, Phys. Rev. B 61, 14607 (2000).

17 F. Coester, Nucl. Phys. 7, 421 (1958); F. Coester and H. Kümmel, ibid. 17, 477 (1960).

18 R.F. Bishop, Theor. Chim. Acta 80, 95 (1991); R.F. Bishop in Microscopic Many-Body Theories and Their Applications, edited by J. Navarro and A. Polls, Lecture Notes in Physics Vol. 510 (Springer-Verlag, Berlin, 1998), p. 1.

19 R.F. Bishop, J.B. Parkinson, and Y. Xian, Phys. Rev. B
43, 13782 (1991); Phys. Rev. B 44, 9425 (1991);

20 R.F. Bishop, R.G. Hale, and Y. Xian, Phys. Rev. Lett. 73, 3157 (1994).

21 D.J.J. Farnell, S.E. Krüger, and J.B. Parkinson, J. Phys.: Condens. Matter. 9, 7601 (1997).

22 C. Zeng, D.J.J. Farnell, and R.F. Bishop, J. Stat. Phys., 90, 327 (1998).

23 R.F. Bishop, D.J.J. Farnell, and C. Zeng, Phys. Rev. B 59, 1000 (1999).

${ }^{24}$ R.F. Bishop, D.J.J. Farnell, S.E. Krüger, J.B. Parkinson, J. Richter, and C. Zeng, J. Phys.: Condens. Matter 12, 6887 (2000).

25 R.R.P. Singh, M.P. Gelfand, and D.A. Huse, Phys. Rev. Lett. 61, 2484 (1988).

26 N.B. Ivanov, S.E. Krüger, and J. Richter, Phys. Rev. B 53, 2633 (1996).

27 C. Gros, W. Wenzel, and J. Richter, Europhys. Lett. 32, 747 (1995).

${ }^{28}$ Z. Weihong, M.P. Gelfand, R.R.P. Singh, J. Oitmaa, and C.J. Hamer, Phys. Rev. B 55, 11377 (1997). 


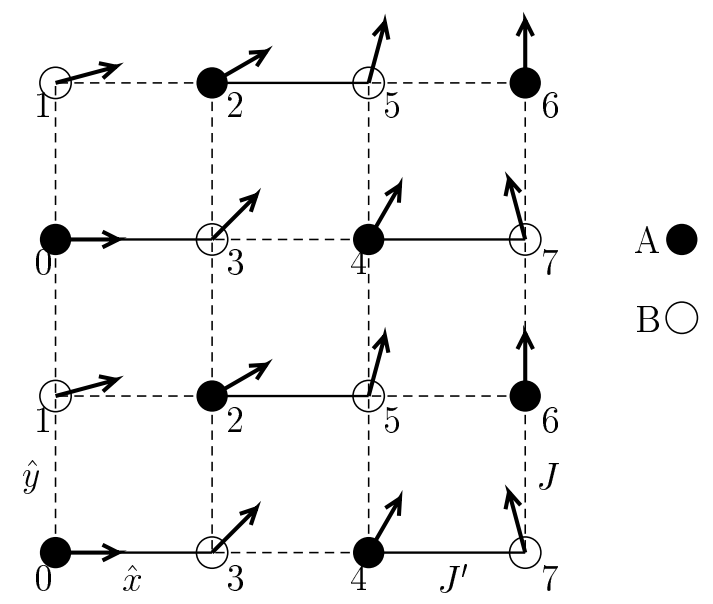

FIG. 1: Illustration of the classical spiral state for the square-lattice Heisenberg model of Eq. (1), with two kinds of regularly distributed nearest-neighbour exchange bonds, $J$ (dashed lines) and $J^{\prime}$ (solid lines). The spin orientations at $A$ and $B$ lattice sites are defined by the angles $\theta_{n}=n \Phi$ where $n=0,1,2, \ldots$, and $\Phi$ is the characteristic angle of the spiral state. The state is shown for $\Phi=\pi / 12$ and $n=0,1, \ldots, 7$ and refers to the ferromagnetic case $(J<0)$ with a $J^{\prime}>|J| / 3$. For the antiferromagnetic case $\left(J>0\right.$ and $\left.J^{\prime}<-J / 3\right)$ all spins on the B sublattice are reversed.

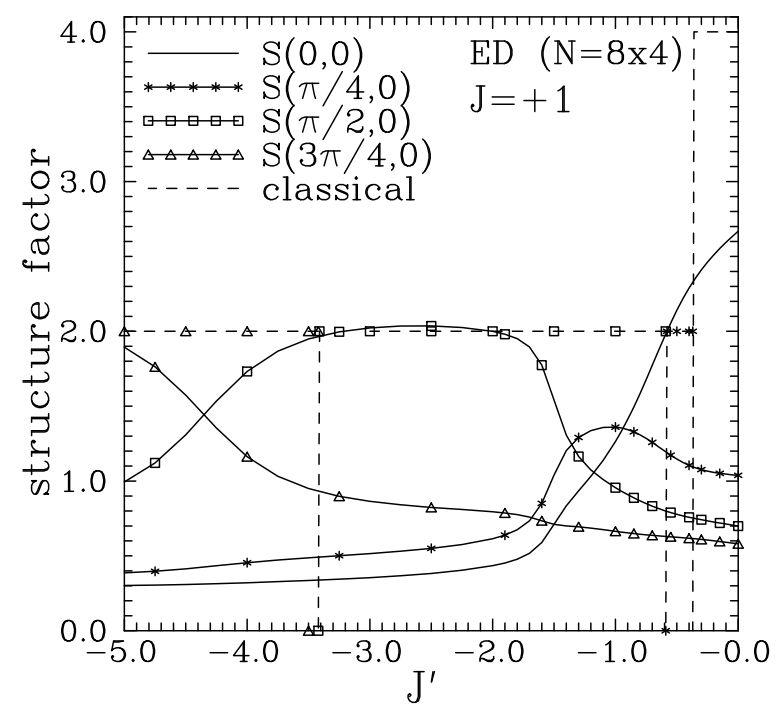

FIG. 2: Ground-state structure factor $S(\mathbf{k}) \propto$ $\sum_{i, j \in A} e^{\mathrm{i}\left(\mathbf{R}_{j}-\mathbf{R}_{i}\right) \cdot \mathbf{k}}\left\langle\mathbf{S}_{i} \cdot \mathbf{S}_{j}\right\rangle$ (i.e., the summation is taken over one sublattice) for a $8 \times 4$ lattice (with antiferromagnetic $J=+1$ ) for the quantum and the classical case for various spiral vectors $\mathbf{k}$. 


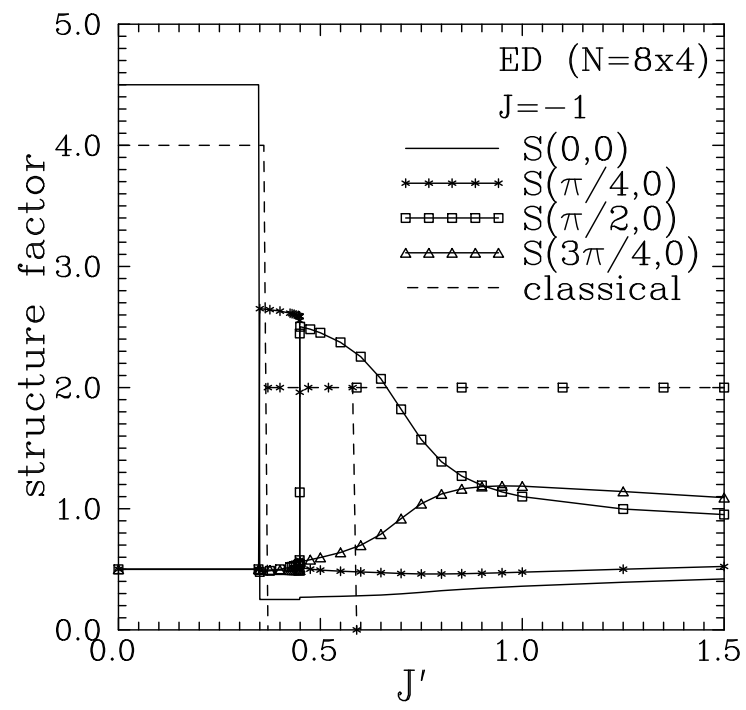

FIG. 3: Ground-state structure factor $S(\mathbf{k})$ (see Fig. 2) for a $8 \times 4$ lattice (with ferromagnetic $J=-1$ ) for the quantum and the classical case for various spiral vectors $\mathbf{k}$.

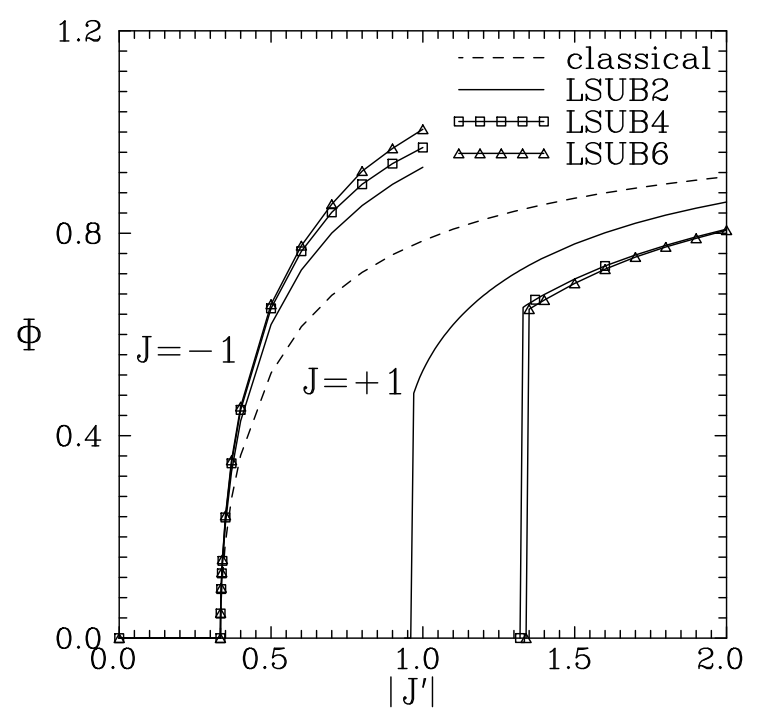

FIG. 4: Pitch angle $\Phi$ versus $\left|J^{\prime}\right|$ for the quantum and the classical case. While $\Phi$ is classically the same for the ferromagnetic case $\left(J=-1, J^{\prime}>0\right)$ and for the antiferromagnetic case $\left(J=+1, J^{\prime}<0\right)$ the quantum pitch angle is different for both cases. The curves left of the classical (dashed) curve belong to $J=-1$ and those right of it to $J=+1$. Note that for the ferromagnetic case $(J=-1)$ for $J^{\prime}>1$ the pitch angle $\Phi$ becomes meaningless, since the spiral order is already destroyed in this region (c.f., Fig. 国). 


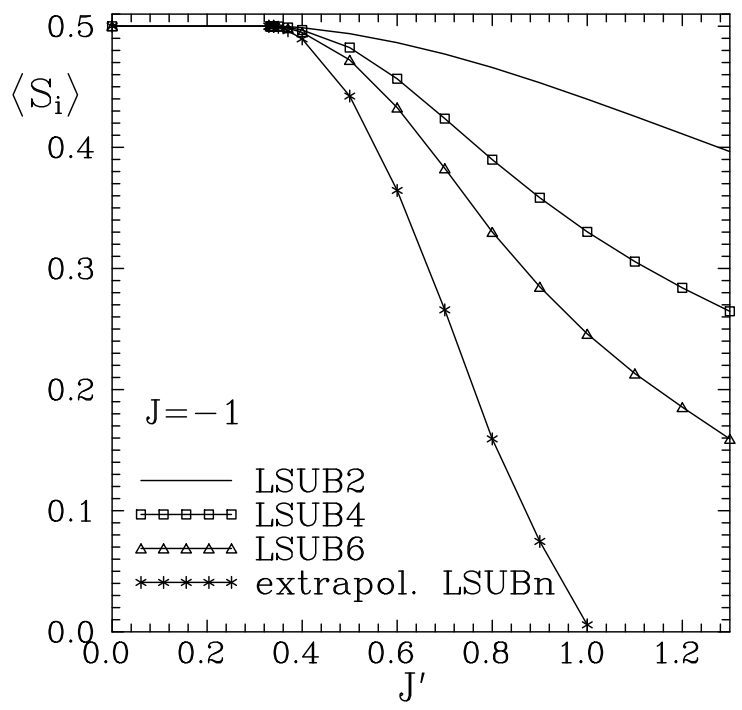

FIG. 5: On-site magnetic moment versus $J^{\prime}$ for the ferromagnetic case $(J=-1)$ calculated within the CCM-LSUB $n$ approximations and extrapolated to $n=\infty$ (The extrapolation is done as described in Ref.16).

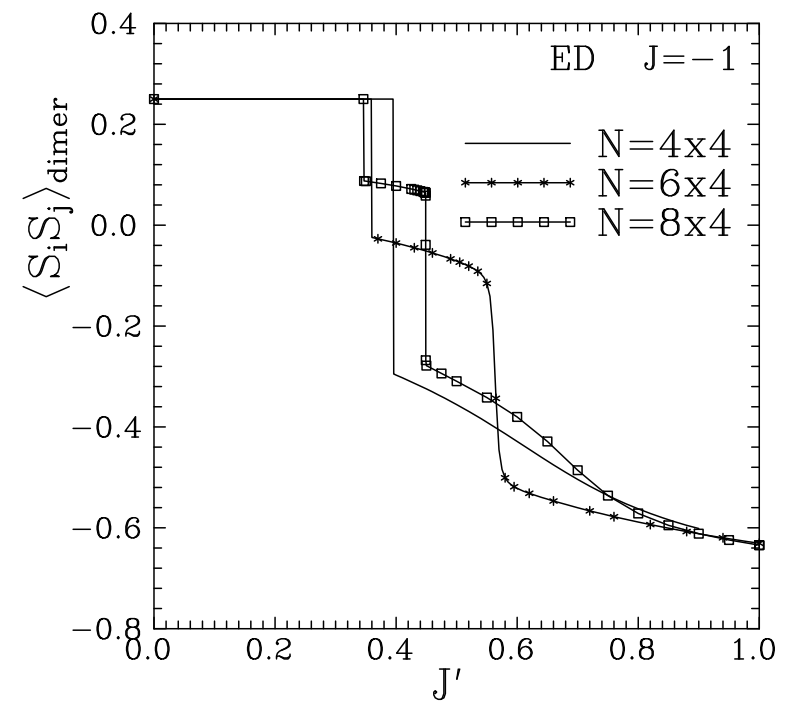

FIG. 6: Nearest-neighbour spin-spin correlation of the two spins connected via a $J^{\prime}$ bond versus $J^{\prime}$ for the ferromagnetic case using exact diagonalization (ED) data. 